\title{
O-33 Characterization of the Fluctuating Detachment State in ASDEX Upgrade
}

\author{
S. Potzel, M. Wischmeier, M. Bernert, R. Dux, H.W. Müller, A. \\ Scarabosio, the ASDEX Upgrade Team \\ Max-Planck-Institut für Plasmaphysik, EURATOM Association, Boltzmannstraße 2, \\ 85748 Garching, Germany
}

\begin{abstract}
In this article we present experimental investigations of divertor detachment in ASDEX Upgrade. For this purpose we performed a series of ohmic and LMode density ramp discharges. The electron density in the divertor volume is determined with a spectroscopic measurement of the Stark broadened line shape of the $\mathrm{D}_{\epsilon}$ line. These measurements are combined with several other diagnostics, yielding a consistent picture of detachment. It will be shown that detachment in ASDEX Upgrade is not a continuously evolving process but can be divided into three different states. Within these states, the conditions in the inner and outer divertor are strongly coupled. Radiative fluctuations, measured with fast diode bolometers, combined with a region of high electron density appear at the X-point during one of these states. We show how $\mathrm{N}_{2}$ seeding during this state alters the divertor conditions. Finally an unstable situation was found, where the divertor plasma oscillates between two detachment states.
\end{abstract}

Key words: ASDEX-Upgrade, Divertor detachment, Power exhaust,

Email address: steffen.potzel@ipp.mpg.de (S. Potzel) 
Impurity seeding

PACS: 52.55.Fa, 52.55.Rk, 52.25.Vy, 52.25.Jm

\section{Introduction}

Avoiding damage of the divertor material by keeping the power load below a certain threshold is a major challenge of future fusion devices such as ITER. For Tungsten, at least in the D-T campaign the foreseen ITER divertor target material, the power load must be kept below $P=5 \mathrm{MW} / \mathrm{m}^{2}$ in continuous operation. This can in ITER only be achieved with the plasma being detached or partially detached $[1,2]$.

Divertor detachment is achieved by a reduction of the temperature in the divertor via increasing the main plasma density or seeding of additional impurities. With a reduction of the temperature, volumetric processes such as charge exchange collisions (below $T_{e} \approx 5 \mathrm{eV}$ ) and recombination (below $T_{e} \approx 1-2 \mathrm{eV}$ ) become dominant. These processes lead to a strong reduction of the ion flux and plasma pressure in front of the divertor target. As a consequence of the pressure reduction and low temperatures close to the target, the region of high electron density is retracted from the target and a knowledge of the electron density distribution in the divertor volume is necessary to understand the detachment process.

As detachment is a 2D strongly coupled nonlinear process a quantitative description is only possible with extensive code simulations. In particular to be able to predict the ITER divertor performance a correct simulation of divertor detachment is indispensable. But even with the most sophisticated codes, divertor detachment of present day machines has not yet been suc- 
cessfully reproduced [3]. Several experimental observations are still not fully understood (e.g. the ion flux asymmetry [4] or flows in the SOL [5]).

In this work, emphasis was put on the evolution of the electron density distribution in the divertor volume, which, especially in the inner divertor, is rather unknown. Furthermore, the detachment process was investigated in the inner and outer divertor simultaneously. The line integrated density measurements were combined with several other divertor diagnostics and a consistent picture of the detachment process is proposed.

\section{Discharge and diagnostic setup}

To study divertor detachment, a series of ohmic and L-mode density ramp discharges has been performed at ASDEX Upgrade, AUG, whose plasma facing surfaces are since 2007 completely covered with tungsten [6]. All discharges were in lower single null divertor configuration with a lower triangularity of $\delta=0.36$, a plasma current of $I_{p}=1 \mathrm{MA}$, a toroidal magnetic field of $B_{t}=2.5 \mathrm{~T}$ and a safety factor of $q_{95} \approx 4$. The electron cyclotron resonance heating, ECRH, power was varied from discharge to discharge. The fuelling species was changed between deuterium and hydrogen and the field direction was changed between forward field (ion $\mathbf{B} \times \nabla \mathbf{B}$ drift towards the lower divertor) and reversed field. After a stable magnetic configuration (separatrix shown in Fig. 1) was established at $1.8 \mathrm{~s}$, the strike point positions where kept constant, because we wanted to measure the time evolution of the divertor plasma excluding any geometric effects. The gas fuelling ramp starts at $1.9 \mathrm{~s}$ and the fuelling was ramped up until the density limit occurred.

Langmuir probes, LP, measure the ion flux, $\Gamma_{D^{+}}$(or $\Gamma_{H^{+}}$respectively), 
and the electron density, $n_{e, t}$, at the divertor targets at the positions shown in Figure 1 with an acquisition time of $\Delta t=0.035 \mathrm{~ms}$. The electron density in the divertor volume, $n_{e, V}$, is determined via a spectroscopic measurement of the Stark broadened $\mathrm{D}_{\epsilon}$ line. Synthetic Stark broadened line shapes, calculated and published by Stehlé [7] based on the model microfield method, are convoluted with the instrument function and the Doppler broadening of a fixed neutral temperature of $T_{n}=5 \mathrm{eV}$. These shapes are fitted to the measured lines in a least square sense, in which $n_{e, V}$ is a fit parameter. For $n_{e, V} \geq 4 \cdot 10^{19} \mathrm{~m}^{-3}$ and $B_{t} \leq 3$. T the Zeeman splitting and variations of $T_{n}$ can be neglected (for details see [8]). This measurement has an uncertainty of $15 \%$, an acquisition time of $\Delta t=2.65 \mathrm{~ms}$ and is a line integrated measurement weighted with the $\mathrm{D}_{\epsilon}$ emissivity along the lines of sight, LOS, which are shown in Figure 1. Fast diode bolometers, AXUV, measure the total radiation between $1 \mathrm{eV}$ and $8 \mathrm{keV}$ [9]. This measurement is not absolutely calibrated and has a sampling rate of $200 \mathrm{kHz}$.

\section{Evolution of divertor detachment - the three detachment states}

We use the ratio of the calculated $\left(\Phi_{D^{+}}^{\text {calc }}\right)$ and measured $\left(\Phi_{D^{+}}^{\text {meas }}\right)$ ion flux to the target as a marker for the magnitude of detachment [10]. Following the simple Two-Point-Model, TPM, [11], in the conduction limited regime the ion flux reaching the divertor target is proportional to the square of the upstream separatrix density $n_{u}^{s e p}$. For Ohmic and L-Mode discharges, $n_{u}^{s e p}$ scales approximately linearly with the line averaged plasma density, $\bar{n}_{e}[12]$. Therefore $\Phi_{D^{+}}^{c a l c}$ is deduced from a interferometer measurement at the plasma edge: 


$$
\Phi_{D^{+}}^{\text {calc }}=C_{1} \cdot\left(n_{u}^{s e p}\right)^{2}=C \cdot \bar{n}_{e}^{2}
$$

The total ion flux to the entire target is obtained by integrating over all Langmuir probe measurements along the divertor surface:

$$
\Phi_{D^{+}}^{\text {meas }} \approx 2 \pi \sum_{\text {iprobes }} \Gamma_{D^{+}}(i) R(i) \cdot\left(\frac{\Delta S(i+1)-\Delta S(i-1)}{2}\right)
$$

The $\Delta S$ coordinate is the distance from the strike point along the divertor surface in poloidal direction, positive values are in the SOL (see Fig. 1). To determine the two constants $C$ in Eq. 1 for the inner and outer divertor, the mean values of $\Phi_{D^{+}}^{\text {meas }}(1.8 \mathrm{~s}<t<1.9 \mathrm{~s})$ before the fuelling ramp are set equal to $\Phi_{D^{+}}^{\text {calc }}$. Simply speaking, when the pressure drops along a field line, then $\Phi_{D^{+}}^{\text {meas }}<\Phi_{D^{+}}^{\text {calc }}$. This ratio is, however, only used as a guide line. It will be shown that the roll over of $\Phi_{D^{+}}^{\text {meas }}$, which we use here as the marker for the onset of detachment, does not necessarily coincide with the condition $\Phi_{D^{+}}^{\text {meas }}<\Phi_{D^{+}}^{\text {calc }}$

We can divide the evolution of detachment in ohmic and L-mode discharges into three different states. The first state, called the onset state, is defined when $\Phi_{D^{+}}$in the inner divertor starts to deviate from the TPM scaling $\left(\Phi_{D^{+}}^{\text {meas }}<\Phi_{D^{+}}^{\text {calc }}\right.$, Fig. 2a). The outer divertor is in the conduction limited regime, i.e. scales with the TPM, throughout this state. The transition to the fluctuating state is defined by the appearance of radiative fluctuations close to the X-point (Fig. 2b,d). Within this state, $\Phi_{D^{+}}$in the inner and outer divertor first increases (in the outer even stronger than the TPM scaling) and then rolls over (Fig. 2a,c). The start of the complete detachment state is defined, on the one hand, when the fluctuations are replaced by broad 
band activities and, on the other hand, by the complete detachment of the inner and outer divertor at the strike point region. At the transition to the complete detachment state, $\Phi_{D^{+}}$in the outer divertor becomes less than the TPM scaling, while its roll over already happened during the fluctuating state (Fig. 2c).

This paper focuses mainly on the divertor plasma conditions during the fluctuating state in forward field direction. A detailed description of the divertor plasma conditions during the three detachment states in forward and reversed field can be found in [13].

\subsection{The radiative fluctuations close to the X-point}

At a certain upstream density, depending on the heating power, there is a sudden onset of a fluctuation band at $f \approx 5.5 \mathrm{kHz}$ (Fig. 2b,d), measured with the AXUV diodes (chords shown in Fig. 3). The width is $\Delta f \approx 3 \mathrm{kHz}$ and the amplitude is about 1.5 times the original radiation level.

Left side of Figure 3 shows the power versus the frequency, averaged over the time interval from $2.4 \mathrm{~s}$ to $2.6 \mathrm{~s}$, for three diodes, where the width and the mean frequency are indicated. By calculating the fluctuation strength for all diodes, the position of these fluctuations can be located. The strength is derived by integrating over the spectrum after subtracting the background level, shown by the red line in Figure 3. The resulting fluctuation strength for all diodes is shown in the right side of Figure 3, where the colour-code represents the strength. It can be seen that the radiative fluctuations are located close to the X-point in the inner SOL.

With hydrogen as fuelling species, the frequency of these fluctuations is $\approx 8 \mathrm{kHz}$, which is roughly the frequency in deuterium times the square root 
of the mass ratio of both species:

$$
8 \mathrm{kHz} \approx \sqrt{\frac{m_{D}}{m_{H}}} \cdot 5.5 \mathrm{kHz}=1.4 \cdot 5.5 \mathrm{kHz}=7.7 \mathrm{kHz}
$$

In reversed field operation with deuterium, there are two frequency bands, one at $f \approx 4.5 \mathrm{kHz}$ and one at $f \approx 9 \mathrm{kHz}$. For all cases, the region of maximum fluctuation strength is in the inner SOL close to the X-point.

\subsection{Divertor plasma parameters}

The target profiles of $n_{e, t}$ and $\Gamma_{D^{+}}$measured by LP and the line integrated $n_{e, V}$ profiles in the divertor volume measured by the Stark broadening diagnostic, $\mathrm{SBD}$, as a function of time are shown in Figure 4 . The $\Delta R$ coordinate for the inner vertical SBD LOS in Figure $4 \mathrm{a}$ is the distance from the X-point along a horizontal line (Fig. 1), the origin is at the X-point position and negative values are in the inner SOL. During the onset state, $\Gamma_{D^{+}}$and $n_{e, t}$ close to the inner strike point roll over, while especially $n_{e, t}$ in the inner far SOL $(\Delta S \approx 14 \mathrm{~cm})$ increases (Fig. $4 \mathrm{~d}, \mathrm{f})$. At the start of the fluctuating state, there is a sudden increase of $\Gamma_{D^{+}}$and $n_{e, t}$ at the inner strike point region and a shift of their peak positions from $\Delta S \approx 1 \mathrm{~cm}$ to $\Delta S \approx 3 \mathrm{~cm}$ (Fig. $4 \mathrm{~d}, \mathrm{f}$ ). During this state, a region of high density with peak values of $\approx 2.5 \cdot 10^{20} \mathrm{~m}^{-3}$, which is an order of magnitude higher than $n_{e, t}$ and $\bar{n}_{e}$, develops in the inner far SOL $(\Delta S \approx 15 \mathrm{~cm}$, Fig. $4 \mathrm{~b})$. In the following these regions are called high density fronts. In addition, a second high density front starts to develop in the inner SOL close to the X-point (Fig. 4a, $\Delta R=-2 \mathrm{~cm}$ ). With the crossed LOS in the inner divertor, the regions where the density is highest can be estimated. The positions of these regions agree well with the total radiation 
distribution. The radiation distribution for $t=2.9 \mathrm{~s}$ is shown in Figure $5 \mathrm{a}$ and the approximated positions of the high density fronts are indicated. The expansion of the density front in the inner far SOL (at $\Delta S \approx 15 \mathrm{~cm}$ ) is consistent with an increase of the ion flux and target electron density in this region (Fig. 4d,f). With increasing upstream density, first the spatial extent of this front grows into the inner SOL and then rolls over. With the start of the complete detachment state, the plasma is completely detached from the inner and outer strike point region $(\Delta S<10 \mathrm{~cm})$. Complete detachment is defined here, when, besides $\Gamma_{D^{+}}$and $n_{e, t}$, also $n_{e, V}$ in front of the target plate is reduced to very low values (Fig. 4). With the SBD measurements in the inner divertor, the movement of the high density front from the target towards and even above the X-point can be monitored, indicated by white arrows in Figure 4a,b. The final position of the density front, at $t=3.5 \mathrm{~s}$, agrees with the radiation distribution (Fig. 5b), which peaks well above the $\mathrm{X}$-point. The high density front in the outer divertor rapidly moves out of the region covered by the SBD (Fig. 4c).

\section{Effect of $\mathrm{N}_{2}$ seeding during the fluctuating state}

In AUG, it is possible to puff impurities below the divertor dome into the plasma in a feedback controlled way [14], which then cool the divertor plasma via line radiation. The amount of injected nitrogen is controlled with a shunt measurement of the thermo-electric current into the outer divertor target, yielding an approximation for the divertor temperature, $T_{t, s h}$ [14].

Such a procedure was applied during the fluctuating detachment state. When the fluctuating detachment state was established, the fuelling gas puff 
and hence the plasma density were kept constant and the $\mathrm{N}_{2}$ seeding was applied. In Figure 6a time traces of the applied $\mathrm{N}_{2}$ puff and the line integrated plasma density are shown. The feedback impurity gas puff starts at $2.5 \mathrm{~s}$ and is controlled such that $T_{t, s h}$ is decreased from $\approx 12 \mathrm{eV}$ to $\approx 2 \mathrm{eV}$. The requested $T_{t, s h}$ and the measured one is shown in Figure 6a. Due to the delay of the $T_{t, s h}$ response on the injected $\mathrm{N}_{2}$ and the feedback controller itself, $\mathrm{N}_{2}$ was puffed intermittently. This is why there are oscillations of $T_{t, s h}$ around the requested value.

With the decrease of $T_{t, s h}$, the frequency of the X-point fluctuations is lowered (Fig. 6c), reaching its minimum of $\approx 1 \mathrm{kHz}$ when the $T_{t, s h}$ oscillations are around the minimal temperature values. Moreover, the densities of the high $n_{e, V}$ fronts in the inner far SOL and X-point region are reduced by $\approx 50 \%$ (Fig. 6b,d) during the phases where $T_{t, s h}$ is close to a local minimum.

\section{Divertor plasma oscillations}

At medium to high densities, with the heating power close to the L-H transition threshold, a situation occurs where the divertor plasma oscillates back and forth between the onset state and the fluctuating detachment state (Fig. 7b,d). The characteristics how the various plasma parameters oscillate are similar to those of the divertor plasma oscillations previously found at JET (compare left side of Fig. 7 with Fig. 4 in [15]). Furthermore, in JET high frequent sub oscillations (with respect to the frequency of the plasma oscillations) in the $D_{\alpha}$ signal where found during one state, as stated in [15]. Compared to the oscillations found here, this is the fluctuating detachment

state where the X-point fluctuations are observed. All together this is a 
strong hint that these divertor plasma oscillations are the same for AUG and JET. As a consequence, the onset and fluctuating detachment states should also have existed at JET.

\section{Summary and discussion}

It was shown that detachment at ASDEX Upgrade is not a continuous evolving process but can be divided into three different states. During the fluctuating state, radiative fluctuations appear. Independent of the field direction, they are located in the inner SOL close to the X-point. Their frequencies, which are between $1 \mathrm{kHz}$ and $8 \mathrm{kHz}$, depend on the mass of the fuelling species and the field direction. Other diagnostics are needed, measuring with such a high sampling rate, to trace these fluctuations back to the electron temperature or to the density in order to understand their origin. High densities, being one order of magnitude higher than the target and the line averaged density, are measured close to the X-point and in the inner far SOL during the fluctuating state. The reason for this most likely increased radial transport remains unknown. There seems to be a connection between the fluctuations and the high density fronts, as with $\mathrm{N}_{2}$ seeding the frequency of the fluctuations as well as the spatial extend and absolute value of the density fronts are reduced. Finally, under certain conditions the divertor plasma was found to oscillate between the onset and the fluctuating state. These observations are similar to the divertor plasma oscillations previously found at JET, leading to the conclusion that these states should also have existed at JET. The experiments in JET where carried out with the open horizontal carbon Mk I divertor, whereas the actual AUG divertor is a closed vertical 
tungsten divertor. If theses states have existed in these JET experiments, then the presented classification of divertor detachment is independent of machine size, divertor geometry and divertor material. Dedicated experiments at different machines, confirming this, must be performed.

\section{References}

[1] ITER Physics Basis, Nucl. Fusion, 39 (1999) 2391.

[2] A.S. Kukushkin, et al., Nucl. Fusion 43 (2003) 716.

[3] M. Wischmeier, et al., J. Nucl. Mater. 415 (2011) S523.

[4] R.A. Pitts, et al., J. Nucl. Mater. 337 (2005) 146.

[5] M. Groth, et al., Nucl. Fusion 49 (2009) 115002.

[6] R. Neu, et al., Phys. Scr. T138 (2009) 014038.

[7] C. Stehlé, et al., Astron. Astrophys. SuppL. Ser. 140 (1999) 93.

[8] S. Potzel, et al., 38th EPS Conference 35G (2011) O2.303.

[9] B. Reiter, et al., 36th EPS Conference 33E (2009) P-1.161.

[10] A. Loarte, et al., Nucl. Fusion 38 (1998) 331.

[11] Keilhacker, et al., Plasma Phys. Contr. Nucl. Fusion Research, (1982) 183.

[12] G.D. Porter, et al., J. Nucl. Mater. 266 (1999) 917. 
[13] S. Potzel, et al., New classification of divertor detachment at ASDEX Upgrade submitted to Nucl. Fusion (2012).

[14] A. Kallenbach, et al., Plasma Phys. Control. Fusion 52 (2010) 055002

[15] A. Loarte, et al., Phys. Rev. Lett. 83 (1999) 3657 


\section{Figure captions}

Figure 1: Lines of sight, LOS, for Stark broadening (blue) and fixed Langmuir probes (red). Also shown is the $\Delta S$ and $\Delta R$ coordinate.

Figure 2: Calculated and measured total ion flux to the inner (a) and outer target (c), line integrated radiated power (b) (measured by V2 in Fig. 3) and its power spectrum (d) of discharge discharge \#27100. The three detachment states (OS), (FS) and (CDS) are marked.

Figure 3: Left: Spectral power versus frequency for three AXUV diodes (black) and fit of an exponential function on the background (red) of discharge \#27100. Right: Fluctuation strength for all AXUV diodes. The colour-code represents the strength.

Figure 4: Horizontal (a) and vertical (b,c) line integrated $n_{e, V}$ profile in the divertor volume and $n_{e, t}(\mathrm{~d}, \mathrm{e})$, and $\Gamma_{D^{+}}(\mathrm{f}, \mathrm{g})$ target profiles in the inner and outer divertor, respectively, of discharge \#27100. The three detachment states are marked.

Figure 5: Total radiation distribution from foil bolometry for two time points of discharge \#27100.

Figure 6: Time traces of (a) requested (black) and measured (blue) $T_{t, s h}, \mathrm{~N}_{2}$ puff (red) and line integrated core plasma density (green); (c) spectrogram of AXUV diode V2. Horizontal (b) and vertical (b) line integrated $n_{e, V}$ profile in the inner divertor volume of discharge \#27326. 
Figure 7: Line integrated plasma density (a), neutral pressure (c) in the inner divertor (red) and private flux (black), $\mathrm{D}_{\alpha}(\mathrm{e})$ in the inner (red) and outer (blue) divertor, line integrated radiated power (b) (measured by V2 in Fig. 3) and its spectrogram (d). 


\section{Figures}

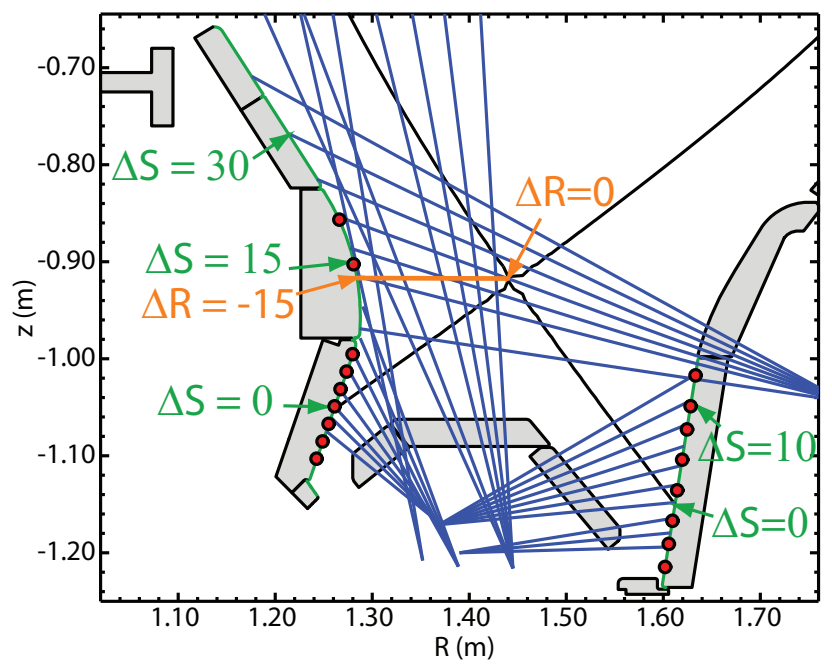

Figure 1: 

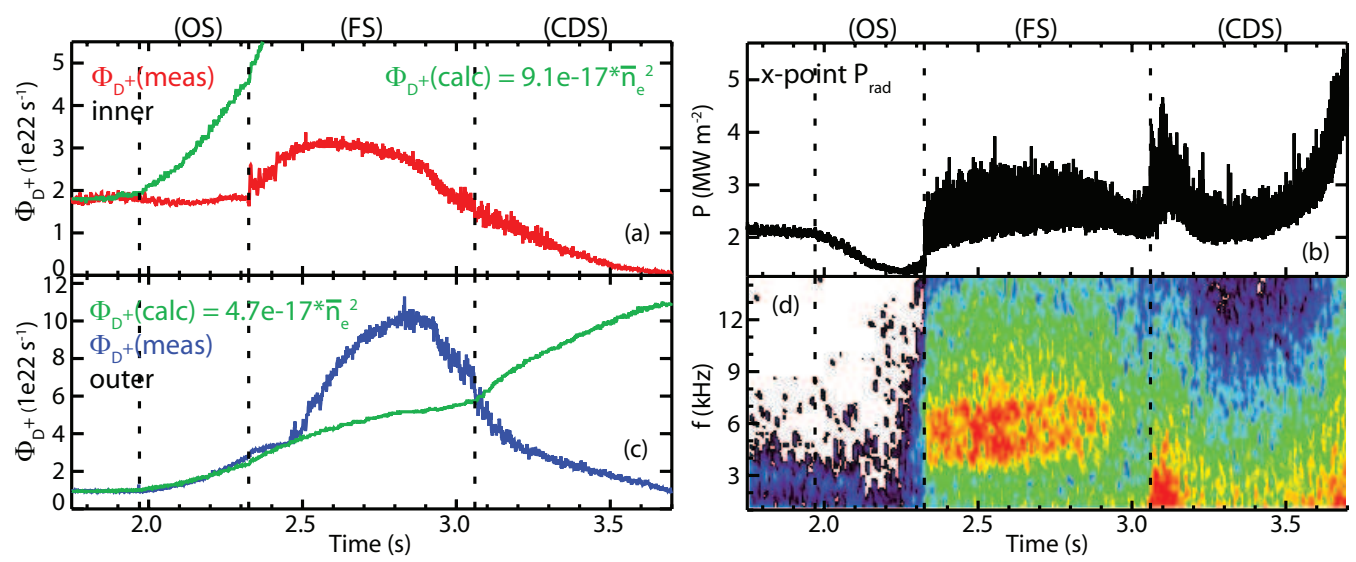

Figure 2: 

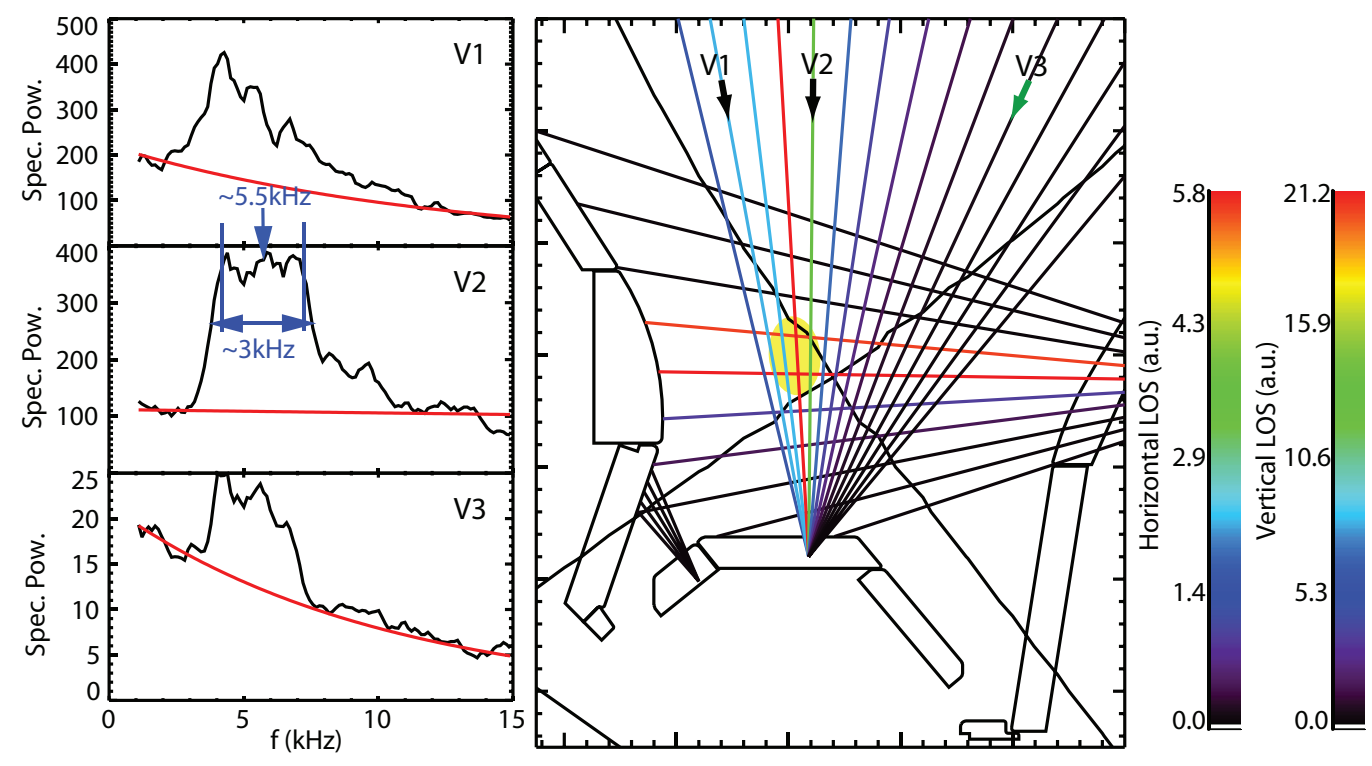

Figure 3: 

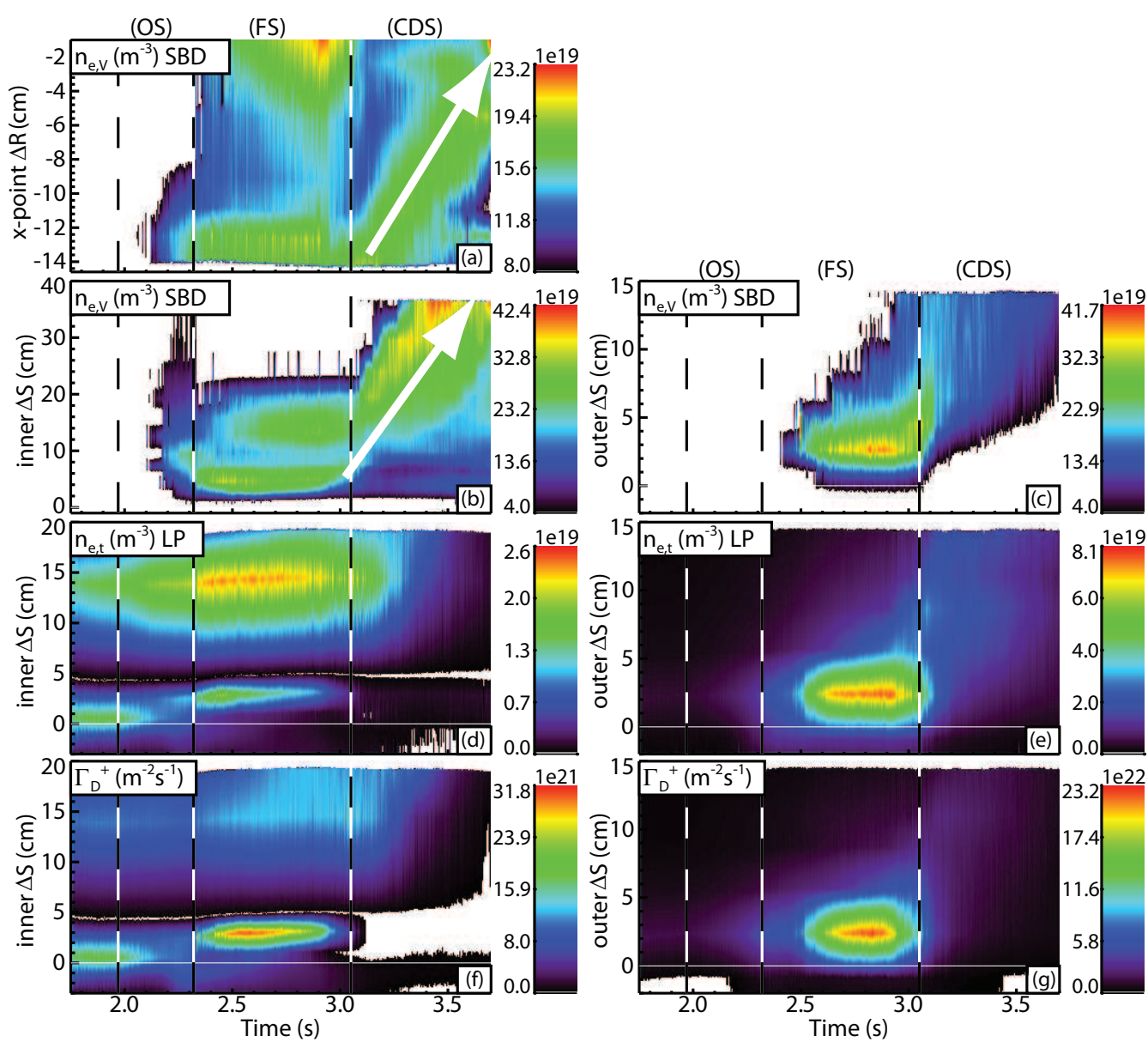

Figure 4: 


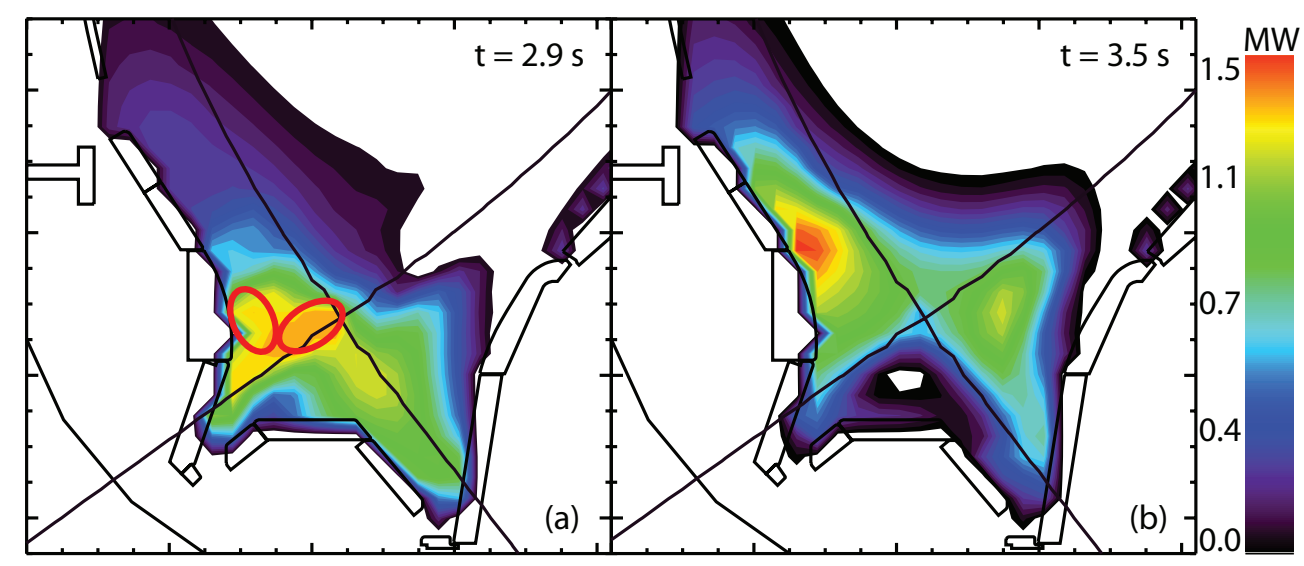

Figure 5: 

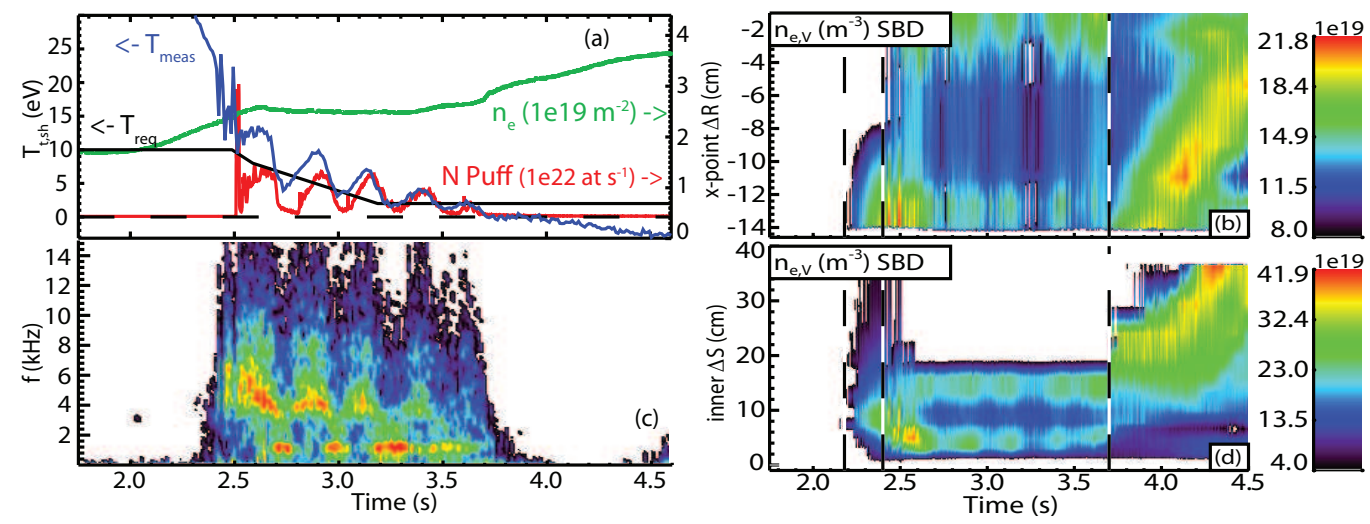

Figure 6: 

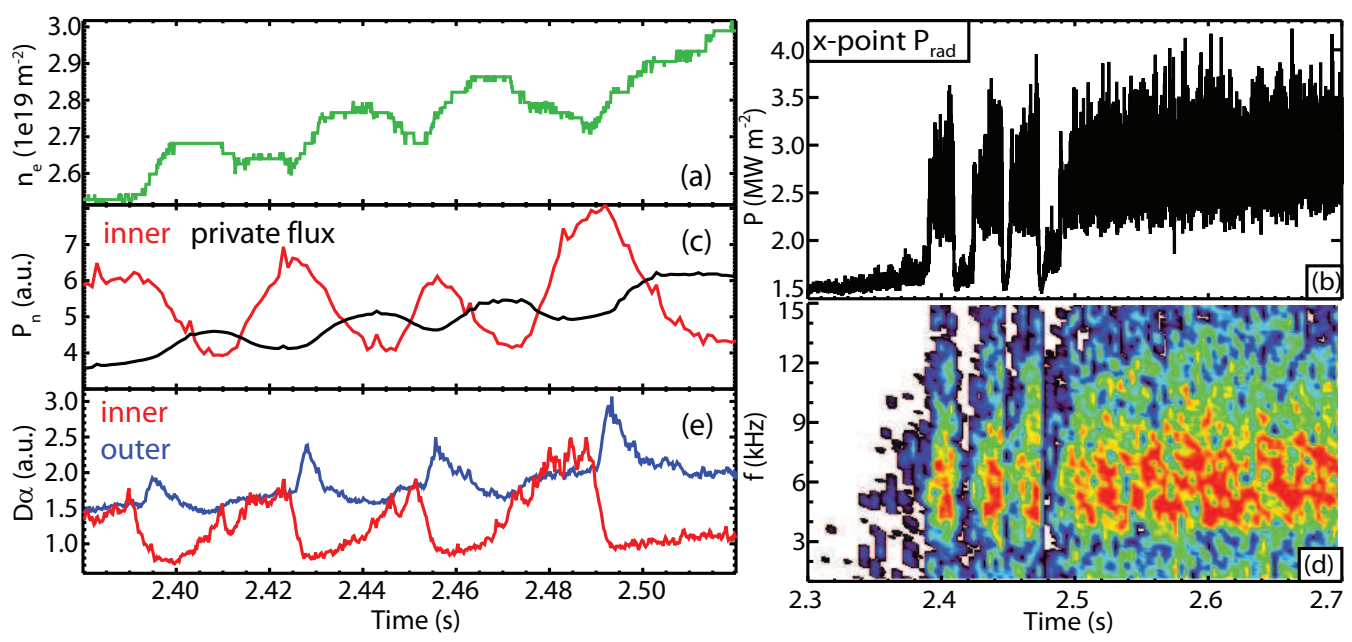

Figure 7: 